\title{
AUTONOMOUS MOBILE ROBOT TEAMS
}

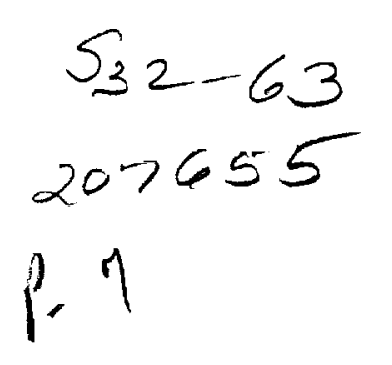

\author{
Arvin Agah \& George A. Bekey \\ Institute for Robotics and Intelligent Systems \\ Computer Science Department \\ University of Southern California \\ Los Angeles, California
}

N94- 30558

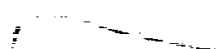

Abstract

This paper describes autonomous mobile robot teams, performing tasks in unstructured environments. The behavior and the intelligence of the group is distributed, and the system does not include a central command base or leader. The novel concept of the Tropism-Based Cognitive Architecture is introduced, which is used by the robots in order to produce behavior, transforming their sensory information to proper action. The results of a number of simulation experiments are presented. These experiments include worlds where the robot teams must locate, decompose, and gather objects, and defend themselves against hostile predators, while navigating around stationary and mobile obstacles.

\section{Introduction}

Teams of robots can be used in a wide variety of applications. Deploying a number of robots in an unknown environment can greatly increase the extent of the area covered for the research mission of planetary explorations, or surveillance of buildings and structures. A team of robots can provide the robustness required in critical missions, where the break down of one unit should not jeopardize the entire mission. The coordination of groups of robots allows them to perform tasks that are too large to be completed by one robot.

A team of robots could function as a centralized group, where the robot that act as the leader can assign sub-tasks to the other robots and monitor and manage the group. In distributed teams, the robots cooperate and perform the task without a leader. Although each type of cooperation has its own advantages, the leadership requirements have the disadvantages of requiring the leader to communicate with all the other robots. Such communications could be costly, and the entire system can come to a halt in the case of the leader's failure to function properly.

This paper describes the study of behavior of a group of distributed robots, surviving and performing tasks in an unstructured environment. We have termed the study of robot team behaviors as Sociorobotics'. In addition to the existence of stationary and mobile obstacles in the world, hostile entities (predators) exit in the world. These predators are mobile and capable of attacking and immobilizing the robots. The world also includes objects of interest to the robots. These objects could be picked up and collected by the robots. If the objects are too large, they must be first decomposed by the robots, before they can be collected. The robots' tasks mainly consist of locating and collecting small objects, locating and decomposing large objects, and locating and attacking predators. These actions are referred to as gather, decompose, and defend, respectively. An example of such tasks is shown in Figure 1. These tasks are performed in the world, while navigating around stationary and mobile obstacles.

Each robot senses and acts upon the world, using a novel architecture, termed Tropism-Based Cognitive Architecture. This architecture is based on the tropisms of the robot, i.e., its likes and dislikes. Such architecture transforms the robot's sensing of the world to potential appropriate actions. The cognitive architecture is tested using simulated robots in an artificial world. This world is similar in its characteristics to an actual world, and the facts and rules of the world are maintained and enforced by the artificial world simulator. The simulator generates an animated world, where the effects of changes inflicted upon the world can be dynamically viewed. In addition, the simulator includes an user-interface for the setting up of the experiments.

The Tropism-Based cognitive architecture enables the robots to survive and function in an unknown world. The desirable feature of such architecture is in its simplicity. Other approaches to cognitive architectures for intelligent systems include the hierarchical structure of intelligence ${ }^{2}$, Subsumption type architectures based on augmented finite state automata ${ }^{9,15}$, neural network based systems $s^{3,5}$, synthetic psychology ${ }^{8}$, reflex action control ${ }^{6}$, and approaches to achieving general intelligence ${ }^{12,14}$. Examples of multiple robot systems include the schemabased navigation ${ }^{4}$, subsumption-based systems ${ }^{16}$, cellular 
robotic systems ${ }^{11,13}$, artificial life systems ${ }^{10}$, and swarm intelligence?.

This paper is organized into six sections. The cognitive architecture is defined in section 2. Certain concepts in sociorobotics are discussed in section 3. Section 4 includes the description of the world, and the world simulator. Section 5 presents a number of performed experiments, and their results. Section 6 contains the conclusions.

\section{Tropism-Based Cognitive Architecture}

The cognitive architecture of each robot is based on the transformation of its sensory information to an action. The architecture will use the concepts of positive and negative tropism ${ }^{17}$. An agent's likes and dislikes will form its perceptions and, therefore, will result in its actions in the Tropism-Based Cognitive Architecture.

The sensing of the entities in the world includes the entity type and the state of the entity. For instance the entity that is sensed could be a predator and the state of the predator could be 'active'. Denoting the set of entities, the set of entity states, the set of robot's actions, and the tropism values by $\left\{\varepsilon_{i}\right\},\left\{\sigma_{i}\right\},\left\{\alpha_{i}\right\}$, and $\left\{\tau_{i}\right\}$, respectively, with $0 \leq \tau_{i} \leq \tau_{\max }$, the tropism values can be represented by a set of relations. In each relation, given the entity and the state of the entity, the robot's action, and the tropism value will be determined.

$$
\{(\varepsilon, \sigma) \rightarrow(\alpha, \tau)\}
$$

In the above example the associated action could be for the robot to attack the predator. The larger the magnitude of the tropism value, the more likely it is for the robot to perform the action.

Once a robot performs a sensory sweep of its surroundings (available sensory area), the set of the tropism values are checked for any matching entity and entity state. For all the matched cases, the selection and the corresponding tropism value is marked. The selection of one action from the chosen set is done by using a biased roulette wheel. Each potential action is allocated a space on the wheel proportional to its tropism values. Then a random selection is made on the roulette wheel, choosing the action. Figure 2 depicts the roulette wheel, where the selection based on the wheel results in the action that is to be performed by the robot. Although currently the tropism values are preset for each robot, work is in progress to have the robots dynamically set these values based on their experiences, i.e., learn. This work is carried out under the research effort called Project Sophia.

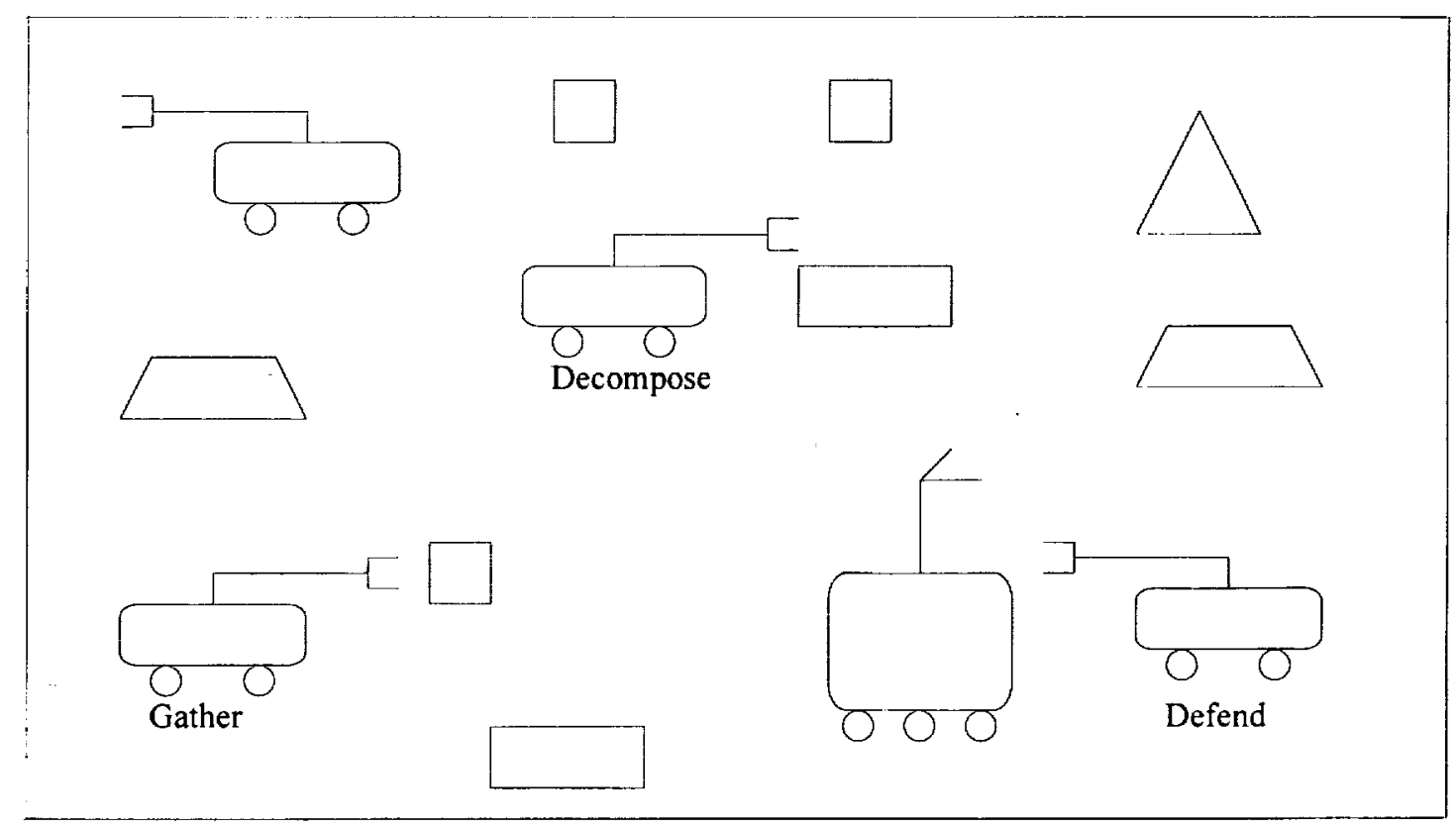

Figure 1: The robot team performing tasks in the world. 


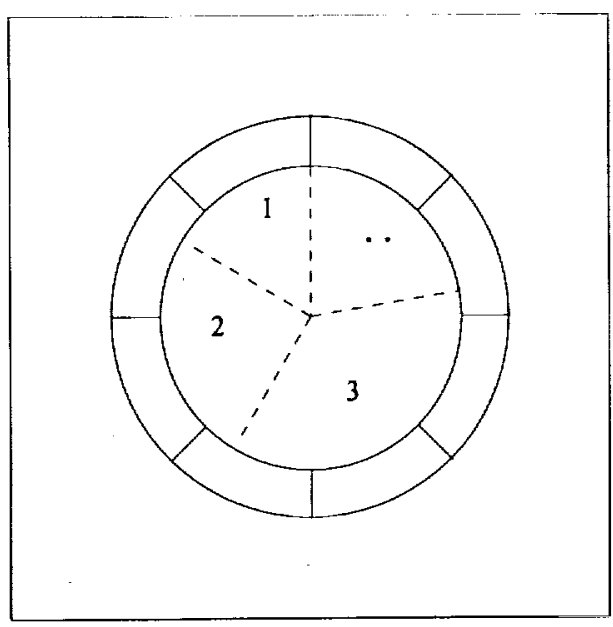

Figure 2: The biased roulette wheel for tropism values.

\section{Sociorobotics}

The study of the behavior of societies of robots is termed sociorobotics. Teams of robots are to survive and perform certain tasks in the world. The performance of the robots as a team is considered, in addition to the individual performance of each robot. Issues pertaining to the task performance of groups of robots are studied as parts of sociorobotics, including: task conditions necessitating a group, environmental factors influencing the group, appropriate group sizes, leadership and its form in a group, structure of a group (including the mixture of specialized versus generalist group members), behavior pattems of the group members, enhancements of group performance, and communication and its format. These concepts are analogous to those of sociobiology ${ }^{18}$.

The parameters that are considered in the study of the behavior of robot teams include: the total elapsed time, the total energy consumption of all the robots, and the difference between the current and the final (desired) world status. The goal is to minimize these values, and by defining the total fitness of the team of the robots as the inverse of these values, the goal is to maximize the fitness function, denoted by $\Phi$. Given the set of entities $Y$, the set of artificial world rules $\Gamma$, the time $T$, the initial and the desired worlds $W, W^{F}$, the set of all robots $\Psi$, and the fitness multipliers $\varphi_{T}, \varphi_{E}$, and $\varphi_{W}$, the fitness function $\Phi$ must be maximized.

$$
\begin{gathered}
\left(Y, \Gamma, W, W^{F}, \Psi, \varphi_{T} \varphi_{E}, \varphi_{W}\right) \Rightarrow \\
\max _{R}\left[\Phi\left(R, \varphi_{T} \varphi_{E}, \varphi_{W}\right)\right]
\end{gathered}
$$

Where $\varphi_{T},{ }_{E}$, and $W$ are fitness multipliers that correspond to the strength of the corresponding time, energy consumption, and world status difference, respectively. Additionally, the role of these multipliers is to convert the units to a scalar. The multiplier $\varphi_{T}$ is of inverse time units and the multiplier $\varphi_{E}$ is in inverse energy units. The multiplier $\varphi_{W}$ is a scalar. The matrix function $\|\ldots\|_{2}$ is the Euclidean norm of the matrix. The addition of 1 to the denominator is to prevent division by 0 . The robot society is considered to be more fit for higher values of the function $\Phi$.

$$
\begin{gathered}
\Phi\left(R, \varphi_{T}, \varphi_{E}, \varphi_{W}\right)= \\
\frac{\varphi_{T}}{1+T}+\frac{\varphi_{E}}{1+\sum_{t=1}^{T} \sum_{\psi=1}^{\rho} \Delta E}+\frac{\varphi_{W}}{1+\left\|W^{F}-W^{T}\right\|_{2}}
\end{gathered}
$$

\section{World \& World Simulator}

The world within which the team of robots reside includes a number of different entity types. These include large and small objects, manipulated by the robots, stationary and mobile obstacles, and mobile predators. The robot is capable of performing action on these entities, as presented in Table 1.

\begin{tabular}{|l|l|}
\hline \multicolumn{1}{|c|}{ Entity } & \multicolumn{1}{c|}{ Action } \\
\hline \hline Space & Move \\
\hline Obstacle & None \\
\hline Base & Enter / Exit \\
\hline Robot & None \\
\hline Other & None \\
\hline Predator & Attack \\
\hline Small Object & Decompose \\
\hline Large Object & Pick / Place \\
\hline
\end{tabular}

Table 1: Entities and the corresponding robot actions.

The world is a two-dimensional space, subdivided into individual blocks that could be occupied by an entity of any type. The center of the world is considered to be the home base of the robots, and the world is divided into eight zones, namely, North, South, East, West, North- 
East, North-West, South-East, and South-West. Figure 3 displays the divided zones of the world.

A robot is capable of sensing and performing action on any of its eight surrounding blocks. The world blocks are enumerated as a two-dimensional matrix, with a row and a column specifying each block. Table 2 includes the block row and columns for the neighboring blocks.

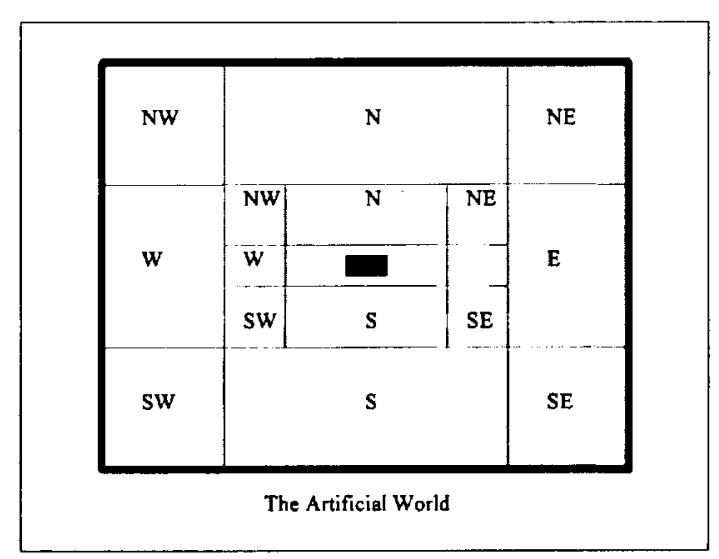

Figure 3: Divided zones of the world.

\begin{tabular}{|l|l|l|}
\hline \multicolumn{1}{|c|}{ Direction } & \multicolumn{1}{c|}{ Row } & \multicolumn{1}{c|}{ Column } \\
\hline \hline $\mathrm{N}$ & row -1 & Column \\
\hline $\mathrm{NW}$ & row -1 & Column -1 \\
\hline $\mathrm{W}$ & row & Column -1 \\
\hline SW & row + I & Column -1 \\
\hline S & row +1 & Column \\
\hline SE & row +1 & Column +1 \\
\hline E & row & Column +1 \\
\hline NE & row -1 & Column +1 \\
\hline
\end{tabular}

Table 2: Row and columns for the eight directions.

The number of zones accessible by a robot decreases once a robot is at the bordering block of the world. By convention it is assumed that the robot is surrounded by stationary obstacles in such cases. For example, once on the very corner of the world, three of the eight blocks are considered to be obstacles. The neighboring blocks of a robot are shown in Figure 4.

The animated display of the world is done using the world simulator. The display of the entities in the world is done in different color schemes. For instance predators are shown in light red, when active. Inactive predators are shown in dark red. Robots are displayed in purple, obstacle in gray and black and objects in blue and green. The world simulator includes the following modules:

- A graphics program for the animated display of the world and its entities.

- A user interface for the administrator to setup and conduct experiments.

- Algorithms to enforce the artificial realities.

- Algorithms to keep track of entity states, including the energy consumption of robots (Each robot consumes energy as it performs a task, proportional to the type of task).

- Algorithms to simulate the cognitive architecture of the robots and to decide the operations of the robots in the world.

The system is implemented on a 80486-based IBMcompatible computers, running Windows 3.1 operating system. The programming is done entirely in $C$ programming language, including the algorithms, the user interface and the graphics. The program is compiled using Quick-C for Windows.

Figures 9 displays the setup screen for an experiment. Figure 10 shows an instance of the world and its robots and other entities. The displayed information include the population of the robots, the total time of the experiment, the total energy consumed by the robots, and the performance of the robot team in terms of gathering, decomposing and defending. The entity at the center of the world is the home base and the larger entities are the large objects.

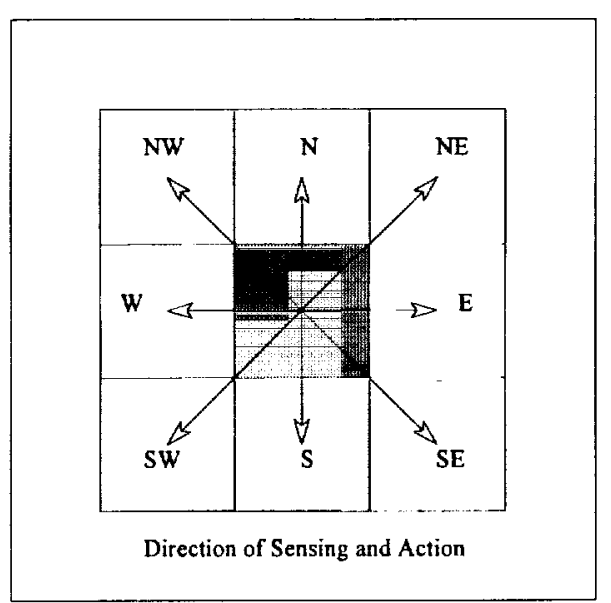

Figure 4: Accessible zones for sensing and action.

\section{Experiments}

Two series of experiments were performed with tames of robots controlled using the Tropism-Based cognitive architecture, using the world simulator. In the first series of experiments, the effects of the stationary 
and mobile obstacles on the energy consumption and performance of the robots were studied. In the second series of experiments, the effects of the robot team size on the energy consumption and performance were investigated.

All the experiments in the first series included 10 robots, 0 predators, 20 large objects, and 20 small objects. The total time of each experiment was 1200 simulation time unit, and the maximum performance achievable was 80 units. The numbers of stationary and mobile obstacles were equal, and their total varied from 0 to 128 obstacles. The graphs for the performance and energy consumption are plotted in Figures 5 and 6, respectively. In all graphs the actual data is in drawn using a solid, thick line, and the fitted curve is done using a dashed, thin line.

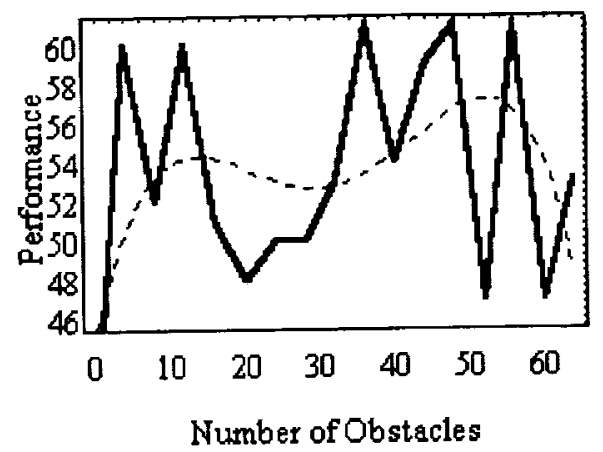

Figure 5: Team performance vs. obstacle density.

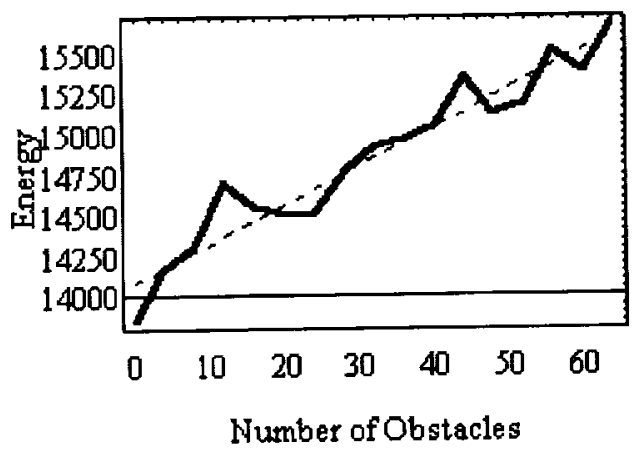

Figure 6: Team energy consumption vs. obstacle density.

As shown, as the number of obstacles increases, the performance increases, although the randomness in the placement of the objects and stationary obstacle results in the non-smoothness of the curve, which is fitted using a degree four polynomial. The energy consumption is linear with respect to the obstacle density, as obstacles result in more energy for the maneuvering.
The experiments in the second series included robot populations from size 0 to teams of 64 robots. The experiment time was set at 700 , with the world including 0 predators, 30 small objects, 30 large objects, 12 mobile obstacles, and 24 mobile obstacles. The total possible performance was 120 units. The graphs for these experiments are shown in Figures 7 and 8.

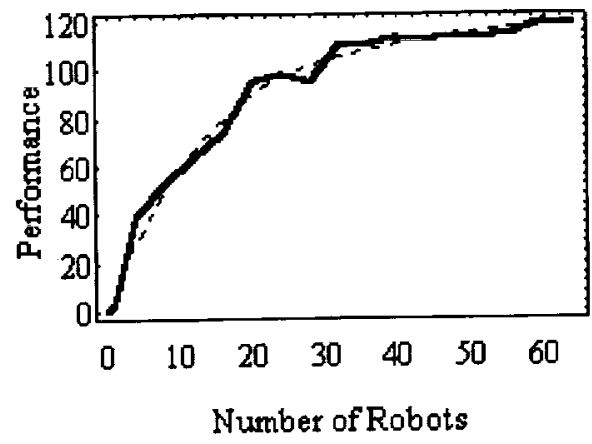

Figure 7: Team performance vs. team size.

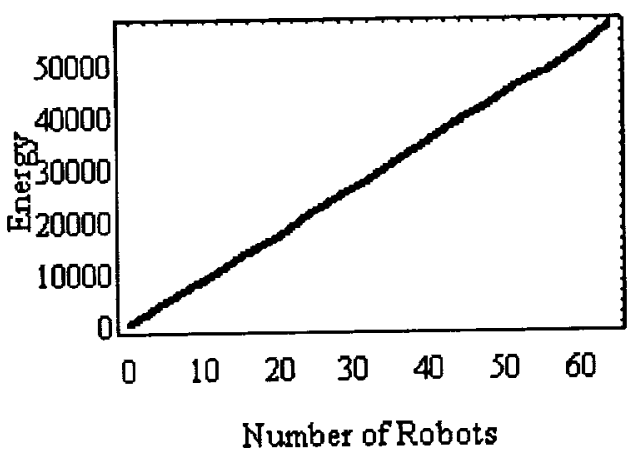

Figure 8: Team energy consumption vs. team size.

In these experiments the performance increases, as more robots are included in the team. The performance eventually levels off since the number of robots reaches a point where the maximum performance in the world is reached. The fitted curve for the performance is a degree four polynomial. The energy increase in the cases of larger teams is linear, since the energy consumptions of all robots are equal. Therefore the size of a robot team can be increased, up to a point where the performance levels off. The faster growth rate of the performance versus the energy consumption justifies the larger team size. 


\section{Conclusions}

A new type of architecture for the control of autonomous mobile robots was presented in this paper. The Tropism-Based Cognitive Architecture is a simple and powerful method for enabling the robots to produce and perform actions based on their sensory input. A team of robots, equipped with this type of architecture was used in a number of realistic simulation experiments. These robots were able to perform a number of tasks, while surviving in a world that contained hostile, mobile predators. The robots located, processed, and collected objects, while navigating around stationary and mobile obstacle in an unstructured world. The work in progress includes a number of extensions to the architecture, and implementing and testing of the concepts on a group of real robots in the physical world.

\section{Bibliography}

[1] Agah, A. (1993). Principles of Sociorobotics. Technical Report IRIS-93-317, Institute for Robotics and Intelligent Systems, University of Southern California, Los Angeles, California.

[2] Albus, J. S. (1991). Outline for a theory of intelligence. IEEE Transactions on Systems, Man, and Cybernetics, 21: 473-509.

[3] Arbib, M. A. (1989). The Metaphorical Brain 2: Neural Networks and Beyond. Wiley-Interscience, New York.

[4] Arkin, R. C. (1992). Cooperation without communication: multiagent schema-based robot navigation. Journal of Robotic Systems, 9: 351-364.

[5] Beer, R. D. (1990). Intelligence as Adaptive Behovior: An Experiment in Computational Neuroethology. Academic Press, San Diego.

[6] Bekey, G. A. and Tomovic, R. (1986). Robot control by reflex actions. In Proceedings of the IEEE International Conference on Robotics and Automation, 240-246.

[7] Beni, G. and Hackwood, S. (1990). The maximum entropy principle and sensing in swarm intelligence. In Varela, F. J. and Bourgine, P. (Eds.) Toward a Practice of Autonomous Systems. MIT Press, Cambridge, Massachusetts, 153-160.

[8] Braitenberg, V. (1984). Vehicles, Experiments in Synthetic Psychology. MIT Press, Cambridge, Massachusetts.

[9] Brooks, R. A. (1989). A robot that walks: Emergent behaviors from a carefully evolved network. Neural Computation, 1: 253-262.

[10] Deneubourg, J. L., Goss, S., Franks, N., SendovaFranks, A., Detrain, C., and Chretien, L. (1991). The dynamics of collective sorting robot-like ants and ant-like robots. In Meyer, J.-A. and Wilson, S. W. (Eds.) From Animals to Animats. MIT Press, Cambridge, Massachusetts, 356-363.

[11] Fukuda, T., Ueyama, T., and Arai, F. (1992). Control strategies for cellular robotic network. In Levis, A. H. and Stephanou, H. E. (Eds.) Distributed Intelligence Systems. Pergamon Press, Oxford, 177-182.

[12] Kaelbling, L. P. (1992). Foundations of Learning in autonomous agents. Robotics and Autonomous Systems, 8: 131-144.

[13] Kawauchi, Y., Inaba, M., and Fukuda, T. (1993). A principle of distributed decision Making of cellular robotic system(CEBOT). In Proceedings of the IEEE International Conference on Robotics and Automation, Vol. 3, 833-838.

[14] Laird, J. E., Newell, A., and Rosenbloom, P. S. (1987). SOAR: An architecture for general intelligence. Artificial Intelligence, 33: 1-64.

[15] Maes, P. and Brooks, R. A. (1991). Learning to coordinate Behaviors. In Iyengar, S. S. and Elfes, A. (Eds.) Autonomous Mobile Robots: Control, Planning, and Architecture, IEEE Computer Society Press, Los Alamitos, California, 224-230.

[16] Mataric, M. J. (1992). Minimizing complexity in controlling a mobile robot population. In Proceedings of the IEEE International Conference on Robotics and Automation, 830-835.

[17] Walter, W. G. (1953). The Living Brain. W. W. Norton \& Company, Inc., New York.

[18] Wilson, E. O. (1980). Sociobiology: The Abridged Edition. The Belknap Press, Cambridge, Massachusetts. 


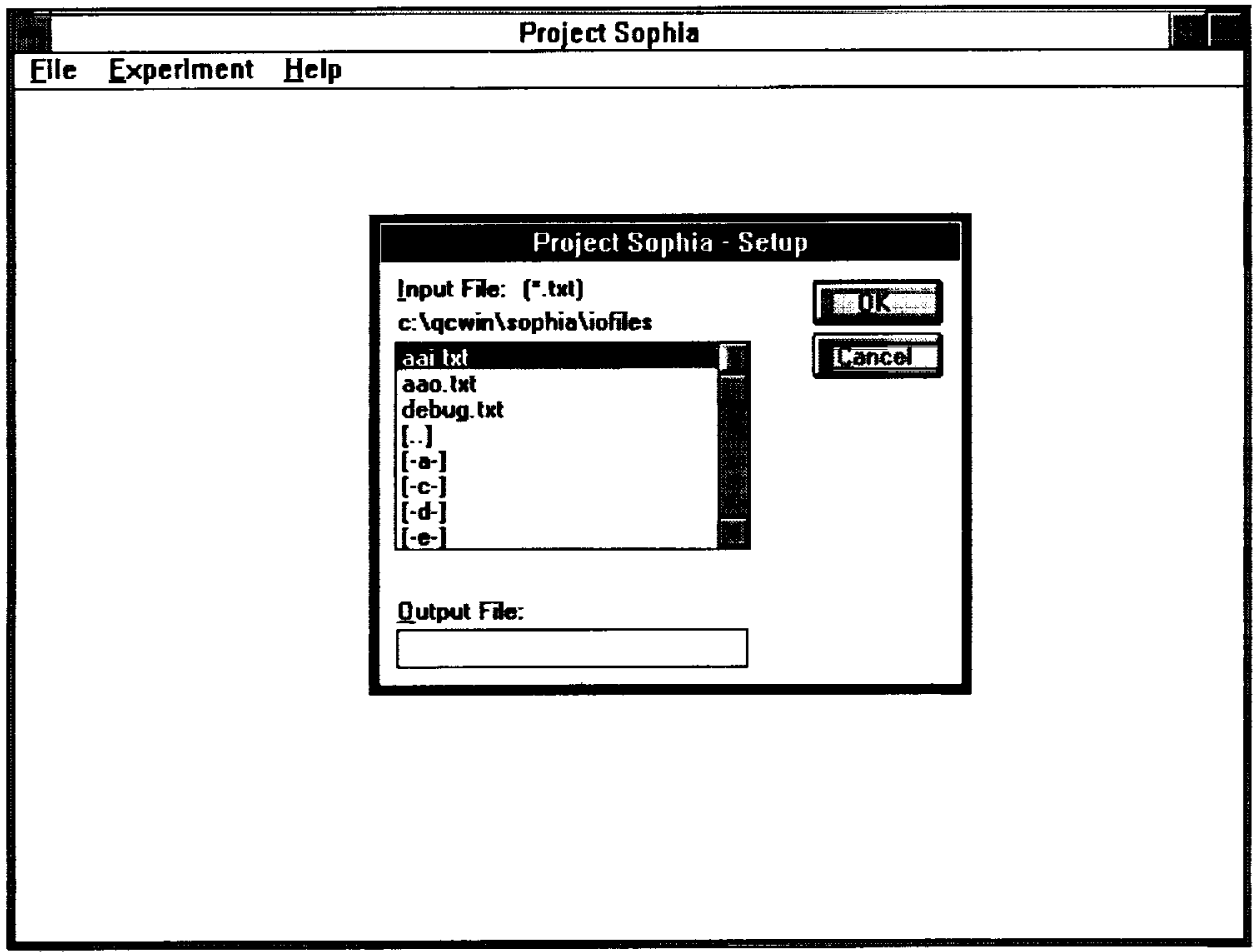

Figure 9: The setup screen for an experiment.

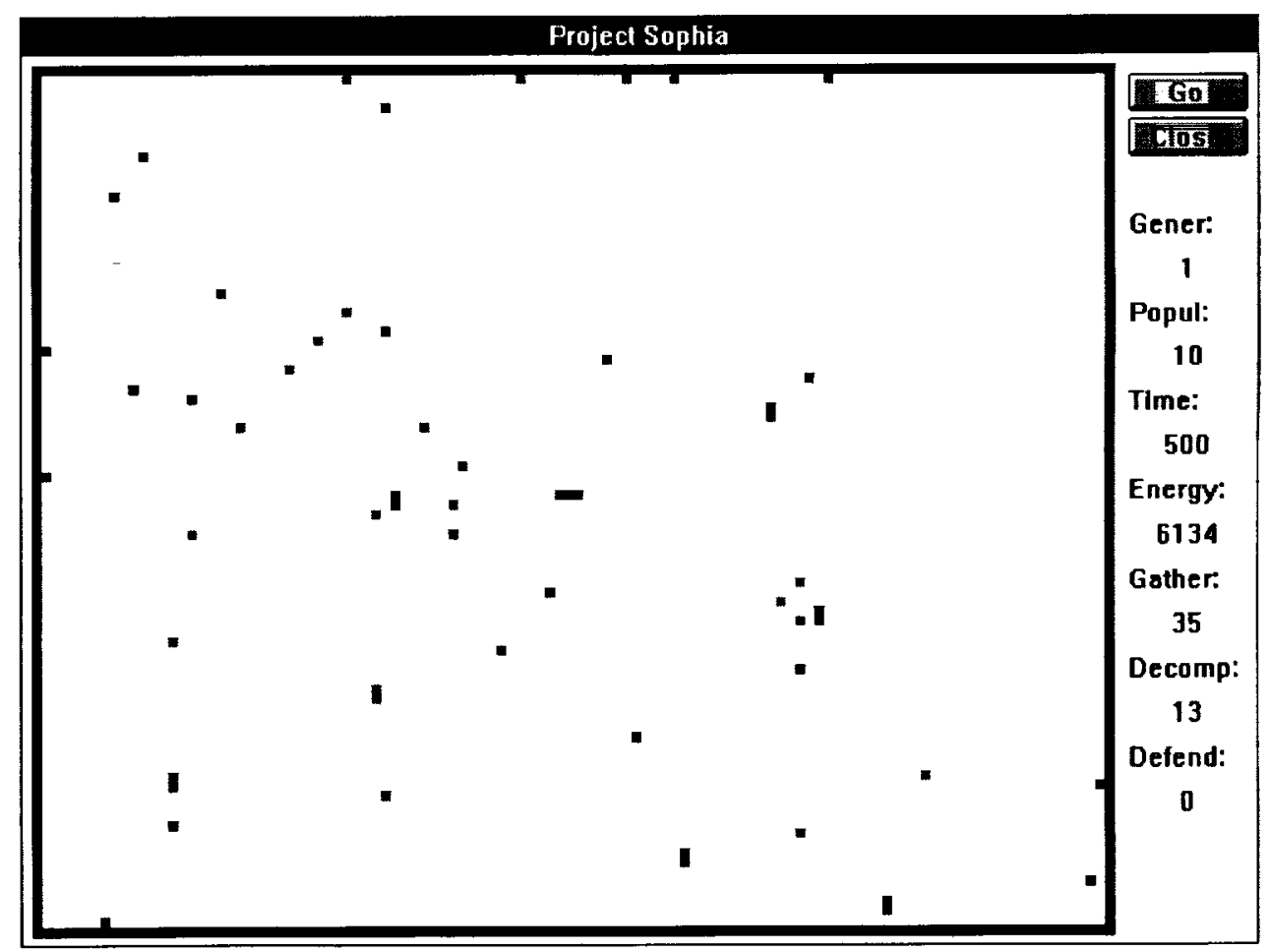

Figure 10: The experiment's world and its entities. 